\title{
¿Superioridad de la amlodipina sobre el enalapril en el tratamiento de pacientes coronarios normotensos?
}

\section{Objetivo}

Evaluar el efecto de amlodipina y enalapril en la incidencia de eventos cardiovasculares en pacientes normotensos con enfermedad coronaria.

Diseño

Ensayo clínico randomizado, doble ciego y controlado con placebo.

Lugar

100 centros de Europa (Francia, Alemania e Italia) Estados Unidos y Canadá

\section{Pacientes}

Hombres (1468) y mujeres (523) de 30 a 79 años derivados para coronariografía con lesiones de más de $20 \%$ y presión arterial (PA) diastólica menor a $100 \mathrm{mmHg}$. Fueron excluidos los pacientes con lesión de tronco superior a $50 \%$, fracción de eyección menor a $40 \%$ y/o insuficiencia cardíaca moderada ó severa.

\section{Intervención}

Los pacientes fueron aleatorizados en tres ramas: 1) 665 a recibir amlodipina (dosis inicial $5 \mathrm{mg}$ y hasta $10 \mathrm{mg}$, si lo toleraban); 2) 657 a recibir enalapril (dosis inicial $10 \mathrm{mg}$ y hasta $20 \mathrm{mg}$, si toleraban); 3 ) 675 a placebo. Todos continuaron tomando el resto de su medicación habitual.

\section{Medición de resultados principales}

El punto final primario fue la incidencia de eventos cardiovasculares en el grupo amlodipina, comparado con placebo (muerte car- diovascular, infarto no fatal, paro cardíaco reanimado, revascularización coronaria, internación por angina inestable, internación por insuficiencia cardíaca, accidente cerebrovascular fatal ó no fatal y nueva enfermedad vascular periférica).

Se realizó además un subestudio angiográfico ( 274 pacientes) para definir la progresión del ateroma medido por eco intravascular (IVUS). El análisis se realizó por intención de tratar.

\section{Resultados principales}

La edad media fue 57 años y $60 \%$ de los pacientes eran hipertensos. La presión arterial aumentó un promedio de $0,7 / 0,6 \mathrm{mmHg}$ en el grupo placebo y disminuyó $4,8 / 2,5 \mathrm{mmHg}$ y $4,9 / 2,4 \mathrm{mmHg}$ con amlodipina y enalapril, respectivamente $(p<0,001$ comparado a ambas drogas con placebo).

La incidencia de eventos cardiovasculares totales fue $23,1 \%$ en el grupo placebo, $16,6 \%$ en el grupo amlodipina y $20,2 \%$ en el grupo enalapril: "Hazard ratio" (HR) amlodipina vs. placebo 0,69 (IC95\% $0,54-0,88 ; p=0,003$ ); amlodipina vs. enalapril 0,81 (IC95\% 0,63$1,04 ; p=0,10)$; enalapril vs. placebo 0,85 (IC95\% 0,67-1,07; $p=0,16)$ El subestudio angiográfico mostró progresión del ateroma con placebo $(p=0,001)$ tendencia a la progresión con enalapril $(p=0,08)$ y no progresión con amlodipina $(p=0,31)$

\section{Conclusiones}

La utilización de amlodipina en pacientes coronarios normotensos reduce los eventos cardiovasculares. El enalapril mostró beneficios menores y no significativos. El uso de amlodipina también mostró reducción de la aterosclerosis coronaria.

Fuente de financiamiento: Laboratorio Pfizer

\section{Comentario}

En los últimos años los bloqueantes cálcicos habían caído en un relativo "desuso" por el aumento del riesgo de eventos en pacientes coronarios tratados con bloqueantes cálcicos de acción corta, fundamentalmente nifedipina. Posteriormente, en especial a partir de la aparición de amlodipina, se retomó el interés en este grupo de drogas. El estudio INVEST ${ }^{1}(n=22576)$ incluyó pacientes hipertensos con enfermedad coronaria, y comparó la estrategia verapamilo-trandolapril con atenolol-hidroclorotiazida, mostrando que los primeros eran tan efectivos como los segundos en la prevención de eventos coronarios, con el beneficio adicional de menor incidencia de diabetes (efecto que podría estar asociado al trandolapril). El estudio $\operatorname{VALUE}^{2}(\mathrm{n}=15245)$ comparó valsartan con amlodipina en hipertensos con alto riesgo cardiovascular, mostrando mayores beneficios en infarto y tendencia a menor accidentes cerebrovasculares (ACV) con amlodipina, y menor incidencia de diabetes y tendencia a reducción de insuficiencia cardíaca con valsartan. El estudio $\mathrm{HOPE}^{3}(\mathrm{n}=9297)$ randomizó pacientes de alto riesgo cardiovascular a recibir ramipril ó placebo y demostró la superioridad del primero en la prevención de eventos cardiovasculares graves. Similares conclusiones dejó el estudio EUROPA $(\mathrm{n}=13655)$ con perindopril.

Vale aclarar que el HOPE y el EUROPA randomizaron cerca de 23000 pacientes entre ambos, mientras que el CAMELOT, que hoy comentamos, randomizó 1997. ¿Pueden sacarse conclusiones de la ausencia de utilidad de los inhibidores de la enzima convertidota de angiotensina (IECA) luego de la gran evidencia a su favor proveniente de estudios anteriores ó se trata de un problema muestral? ¿Existe un efecto de clase ó no son iguales enalapril, ramipril y perindopril? ¿Es realmente mejor amlodipina ó enalapril? No creo que tengamos aún la respuesta definitiva. Podríamos decir que los inhibidores de la enzima de conversión (IECA) los antagonistas del receptor de angiotensina (ARA) y ahora los bloqueantes cálcicos (en particular la amlodipina) tienen beneficios en el tratamiento de pacientes coronarios y/ó vasculares de alto riesgo.

\section{Conclusión del comentador}

Hasta que tengamos más evidencia, todas estas drogas pueden ser beneficiosas y deberemos evaluar cuál es la mejor estrategia en nuestro paciente individual. Más allá de la droga elegida, es importante destacar que estos enfermos parecen constituir un grupo similar a los diabéticos y los pacientes con insuficiencia renal, donde el tratamiento agresivo de la presión arterial está justificado, con el objetivo de lograr cifras tensionales inferiores a las de los pacientes de bajo riesgo cardiovascular.

Mariano Falconi [ Especialista en Cardiología. Servicio de Cardiología del Hospital Italiano de Buenos Aires. ]

Falconi M. ¿Superioridad de la amlodipina sobre el enalapril en el tratamiento de pacientes coronarios normotensos.? Evid. act. pract. ambul. 9(1):6.EnFebr.2006. Comentado de: Nissen S, Murat Tuzcu E, Libby P y col. Effect of Antihypertensive Agents on Cardiovascular Events in Patients With Coronary Disease and Normal Blood Pressure (The CAMELOT Study). JAMA. 2004 Nov 10;292:2217-2226. PMID: 15536108.

\section{Referencias}

1. A calcium antagonist vs a non-calcium antagonist hypertension treatment strategy for patients with coronary artery disease. The International VerapamilTrandolapril Study (INVEST): a randomized controlled trial. Pepine C, Handberg E, Cooper-DeHoff R y col. JAMA, 2003; 290: 2805-2816

2. Outcomes in hypertensive patients at high cardiovascular risk treated with regimens based on valsartan or amlodipine: the VALUE randomised trial. Julius S, Kjeldsen S, Weber M, y col. Lancet. 2004;363:2022-31

3. Effects of an angiotensin-converting-enzyme inhibitor, ramipril, on cardiovascular events in high-risk patients. The Heart Outcomes Prevention Evaluation Study Investigators. Yusuf S, Sleight P, Pogue J y col. J Med. 2000 Jan 20;342(3):145-53.

4. Efficacy of perindopril in reduction of cardiovascular events among patients with stable coronary artery disease: randomised, double-blind, placebo-controlled, multicentre trial (the EUROPA study). Fox K. Lancet 2003;362:782-788 